\title{
Research Paper \\ Comparing the Radioprotective Effects of Brewed Rosa damascena and Vitamin E on Ionizing Radiation-Induced Chromosomal Aberrations in Human Peripheral Blood Lymphocytes Using Micronucleus Assay in Binucleated Cells
}

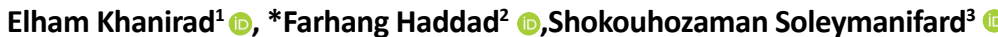

1. Department of Biology, Faculty of Sciences, Islamic Azad University Mashhad Branch, Mashhad, Iran.

2. Department of Biology, Faculty of Sciences, Ferdowsi University of Mashhad, Mashhad, Iran.

3. Department of Medical Physics, Faculty of Medicine, Mashhad University of Medical Sciences, Mashhad, Iran.

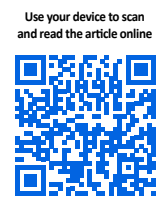

citation Khanirad E, Haddad F, Soleymanifard Sh. [Comparing the Radioprotective Effects of Brewed Rosa damascena and Vitamin E on lonizing Radiation-Induced Chromosomal Aberrations in Human Peripheral Blood Lymphocytes Using Micronucleus Assay in Binucleated Cells (Persian)]. Quarterly of "The Horizon of Medical Sciences". 2020; 26(1):14-23. https://doi.org/10.32598/ hms.26.1.2979.1

Key words:

Rosa damascena, Vitamin E, X-radiation, Micronucleus, Lymphocyte

\section{A B STRACT}

Received: $25 \operatorname{Jan} 2019$ Accepted: 10 Sep 2019 Available Online: 01 Jan 2020

Aims For occupational and therapeutic reasons, many people are exposed to the harmful effects of lonizing Radiation (IR) including Chromosomal Aberrations (CA) every day. Radioprotective agents are able to reduce these damages through mechanisms such as scavenging free radical, donating hydrogen to damaged molecules and increasing activity of antioxidant enzymes. Medicinal plants, traditionally used in different societies, have special advantages due to their low side effects and cost-effectiveness compared to the chemical radioprotectors. Rosa damascena is one of these plants that is widely used in traditional medicine. The aim of this study was to investigate the radioprotective effects of brewed Rosa damascena in comparison with Vitamin $\mathrm{E}$.

Methods \& Materials In this experimental study, the radioprotective effect of 1-week use of brewed Rosa damascena on the CA induced by $2 \mathrm{~Gy} I \mathrm{R}$ in comparison with vitamin $\mathrm{E}$ in peripheral blood lymphocytes of 10 volunteers, 1,24 , and $96 \mathrm{~h}$ as well as one week after the last intake was investigated using binucleated cell micronucleus assay.

Findings The use of brewed Rosa damascena $1 \mathrm{~h}$ after the last intake could significantly reduce the frequency of micronuclei. This result was similar to the effect of vitamin $E$ at the same time.

Conclusion Brewed Rosa damascena is able to protect cells from IR-induced damages and can be used as a cheaper radioprotector with the possibility of daily use compared to vitamin $\mathrm{E}$.

\section{Extended Abstract}

\section{Introduction}

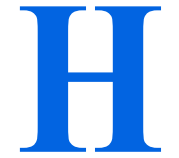

umans are exposed to oxidizing agents, including Ionizing Radiation (IR) everyday for a variety of reasons. The destructive effect of IR on living cells and tissues has already been proven [1]. IR exerts its most destructive effect indirectly by affecting water molecules and producing free proximal and hydroxyl radicals. Biological systems, with their antioxidant defense systems, are somewhat protected against the potentially harmful effects of free radicals [2]. Several studies have shown that the use of IR increases the frequency of micronucleus in peripheral blood lymphocytes [3-6].

\section{* Corresponding Author:}

Farhang Haddad, PhD.

Address: Department of Biology, Faculty of Sciences, Ferdowsi University of Mashhad.

Tel: +98 (915) 3114330

E-mail: haddad@um.ac.ir 
Due to the widespread use of IR in diagnostic and therapeutic purposes, many efforts are being made today to find radiation protectors with minimal side effects [7]. Vitamin E and its derivatives have long been recognized as radiationprotective agents that can reduce the destructive effects of IR by collecting free radicals caused by oxidative stress [8, 9]. Due to the destructive effects of IR and its widespread use, it is necessary to find radioprotectors with low side effects. This study aimed to investigate the radioprotective effects of brewed Rosa damascena in comparison with Vitamin E.

\section{Materials and Methods}

This is an experimental study conducted on peripheral blood lymphocytes collected from 10 human samples (aged 20-25 years with a diet without antioxidants or low in antioxidants, and no history of smoking) in the genetic laboratory of the Department of Biology, Ferdowsi University of Mashhad. Participants were selected from among the volunteers who filled out a questionnaire on lifestyle and general health. They were randomly divided into two groups of Vitamin $\mathrm{E}(\mathrm{n}=5)$ and Rosa damascena $(\mathrm{n}=5)$. The first group was given one vitamin E $200 \mathrm{mg}$ capsule (Dana Pharmaceutical Co.) for one week at 10:00 AM. The second group was given a glass of brewed Rosa damascena for one week at 10:00 AM.

To prepare each glass of brewed Rosa damascena, 1 gram of dried Rosa damascena (Gol Kuh Company) was placed in $250 \mathrm{ml}$ of boiling water for 10 minutes. Blood sampling of volunteers was collected once before consumption of vitamin E/Rosa damascena and four times 1,24,96 h and one week after the last intake in heparin tubes and then cultured for $72 \mathrm{~h}$. The cells were harvested using cytochalasin $\mathrm{b}$ and then, the frequency of micronuclei was calculated.

\section{Results}

X-radiation with a dose of 2 Gy significantly increased the frequency of micronucleus in binucleated cells (9.33\%) compared to controls $(0.132 \%)(\mathrm{P}<0.05)$, (Figure 1$)$. Micronuclei frequency in binucleated cells of volunteers who used $200 \mathrm{mg}$ vitamin $\mathrm{E}$ for one week was decreased significantly only $1 \mathrm{~h}$ after the last vitamin intake compared to the exposed group received no vitamin $\mathrm{E}(\mathrm{P}<0.05)$. After $1 \mathrm{~h}$, there was no decrease in the micronuclei frequency (Figure 1). The use of brewed Rosa damascena could significantly reduce the frequency of micronuclei induced by IR only 1 $\mathrm{h}$ after the last intake $(\mathrm{P}<0.05)$ compared to the exposed group received no brewed Rosa damascena. After $1 \mathrm{~h}$, there was no decrease in the micronuclei frequency (Figure 1).

\section{Discussion}

In other studies, IR increased the frequency of micronuclei in peripheral blood lymphocytes at a level similar to that of the present study [3-6]. In the study by Rostami et al. [8], Vitamin E was also able to reduce the frquency of macronuclei in peripheral blood lymphocytes induced by $\mathrm{X}$-rays only $1 \mathrm{~h}$ after use. The results of the present study show that brewed Rosa damascena can be used as a suitable radiation protector in people exposed to IR for various reasons shortly after consumption. Brewed Rosa damascena has long been used as a drink with a protective effect similar to vitamin E. Therefore, it can be used daily as an available brew by people who are particularly exposed to unwanted IR. To investigate the more effective protective effect of this plant, its phenolic compounds such catechin and flavonoids can be used in pure form. Impossibility to use more volunteers and also generalization of the study to plants of the same family due to the increase of the study groups were some of the limitations of our study.

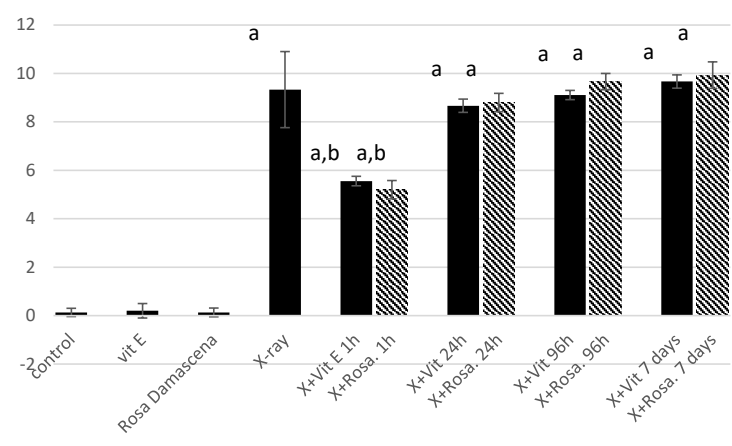

Quarterly of

The Horizon of Medical Sciences

Figure 1. Comparing the protective effects of vitamin $\mathrm{E}$ and brewed Rosa damascene against 2 Gy IR. a significant compared to control group; $\mathrm{b}$ significant compared to the exposed groups $(\mathrm{P}<0.05)$ 


\section{Ethical Considerations}

Compliance with ethical guidelines

This study was approved by Iran National Committee for Ethics in Biomedical Research (Code: 21272).

Funding

This research did not receive any specific grant from funding agencies in the public, commercial, or not-forprofit sectors.

Authors' contributions

All authors contributed in preparing this article.

Conflicts of interest

The authors declared no conflict of interest.

Acknowledgements

The authors would like to thank Islamic Azad University of Mashhad Branch, Department of Biology at Ferdowsi University of Mashhad, and the genetic laboratory staffs of the Department for the valuable cooperation and support. 


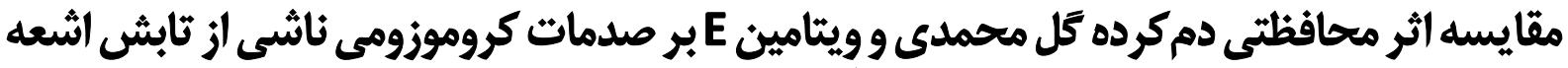

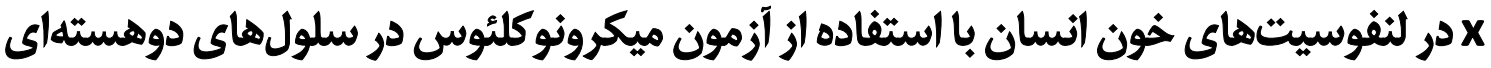

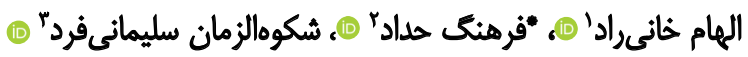

1. كروه زيستشئاسي، دانشكده علوم، داتشكاه آزاد اسلامي واحد مشهبد، مشهبه، ايران.

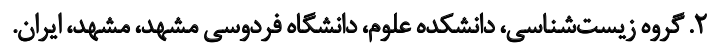

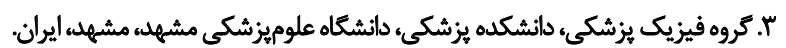

\section{OS}

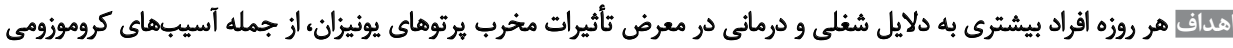

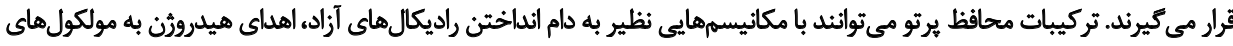

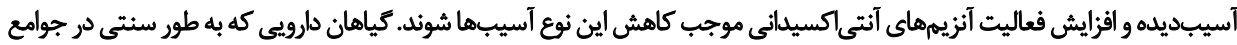

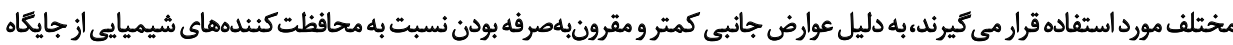

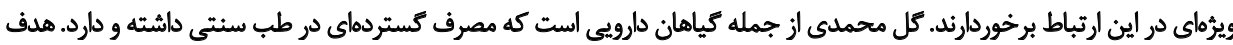
اين مطالعه بررسى اثرات ضدير ترئوي اين كياه است.

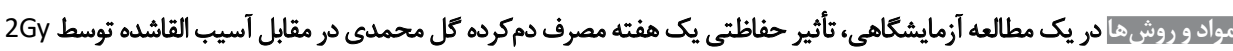

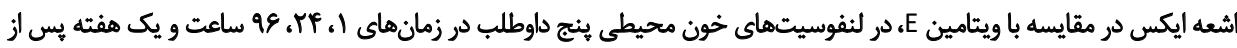

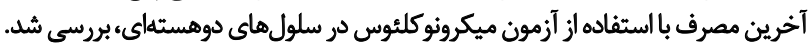

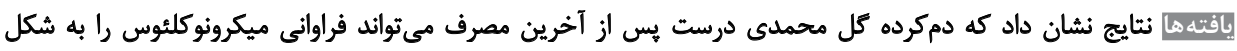

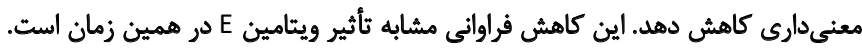

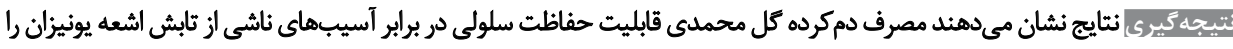

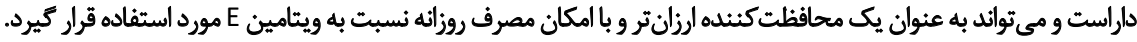

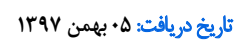
تاريخ هذيرش: 19 شهريور

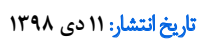

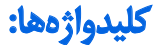

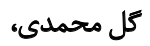

ويتامين E، اشعه مئه

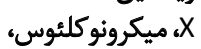
لنفوسيت

سيستمهاى بيولوزيك،بادا داشتن سيستمهاى دفاع آنتىاكسيدانى،

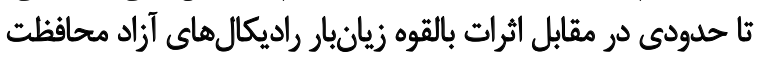

daleo

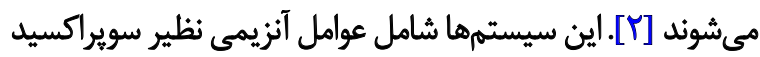

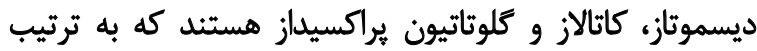

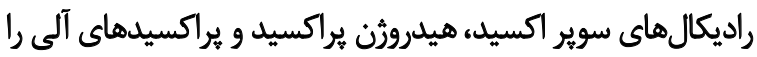

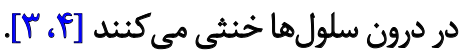
در شرايط عادى، اغلب بين توليد راديكالهاي آزاد و سيستم

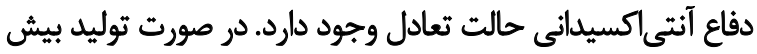

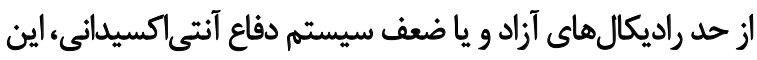

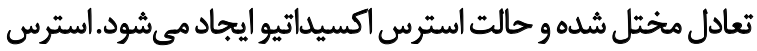
اكسيداتيو شديد مىتواند منجر به القاي نإيايدارى رُنومى و در نتيجه

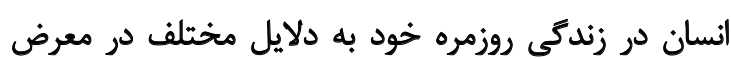

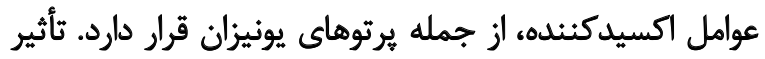

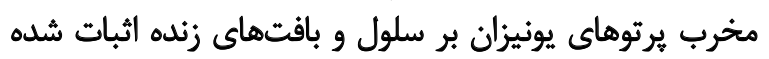

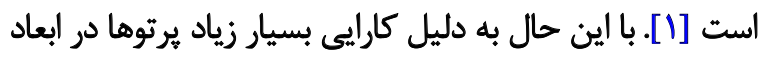

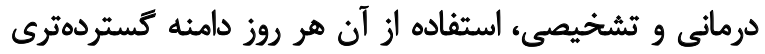

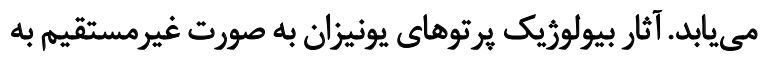

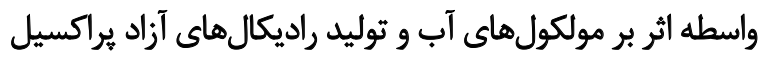

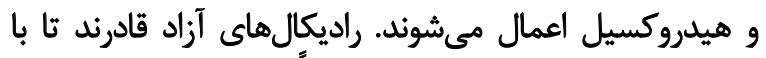

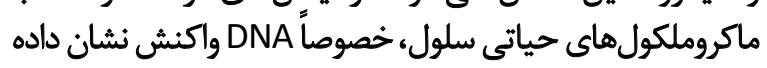
و صدمات جدى راو وارد كثند. 
كه جهت تسهيل استفاده به صورت جاى كيسهاي ساخته شده

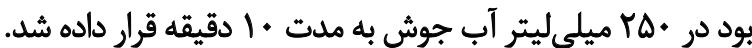

\section{حْونَيَي}

خونكيرى از داوطلبان يكبار قبل از مصرف دمكرده و يا

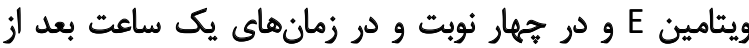

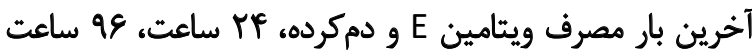

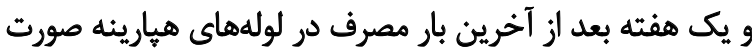

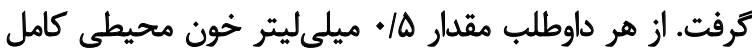

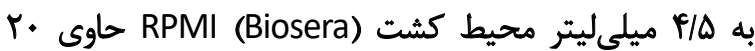

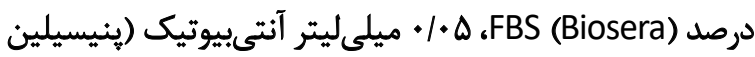

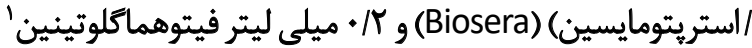
(Gibco)

تيمار با الشعه

محيطهاي كشت تهيهشده از هر داوطلب در فلاسكهاي كشت

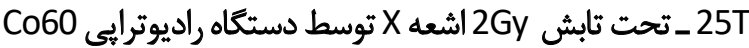

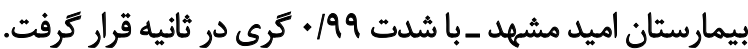

\section{بوداشت سلولى}

به محيطهاى سلولى fA ساعت ڤيس از شروع كشت،

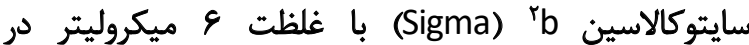

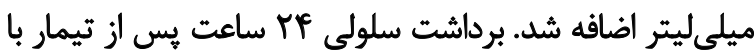

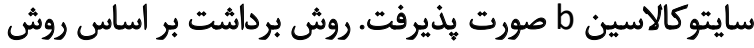

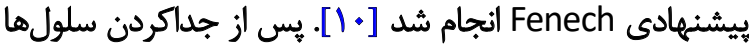

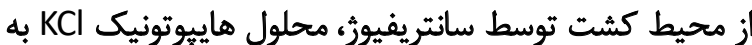

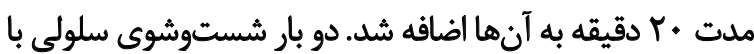

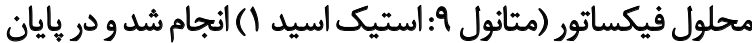

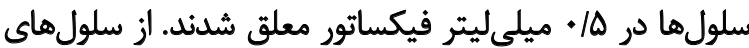

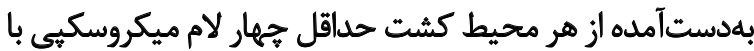
يرتاب كردن جند قطرهاز سوسيانسيون سلولى حاصله تهيه شد.

\section{رونَّآميزى و شمارش سلولى}

رنكَآميزى لامها با استفاده از كيمساى • ا درصد و در مدت

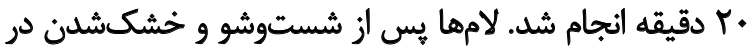

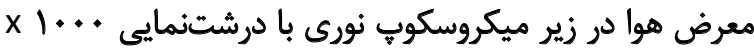

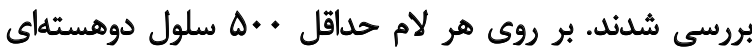

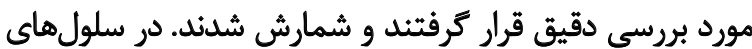

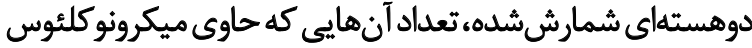
بودند مشخص شد. فراوانى ميكرونوكلئوس در هر لام از از فرمول

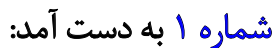

1. Phytohaemagglutinin (PHA)

2. Cytochalasin B
[آسيبهاي سلولى شود [ه]]. بنابراين در اين شرايط موجودات زنده نياز به تركيبات محافظتى علاوه بر سيستمهاي موجود دارئن دارند. تركيبات محافظ يرتو مي توانئد با مكانيسمهايى نظير به

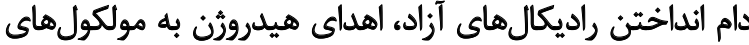

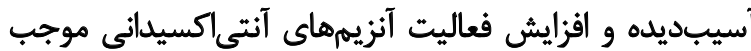

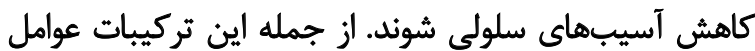
حاوى كوكرد و ويتامين هايى نظير E و C و و فلاونوئيدها هستيند

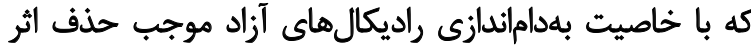

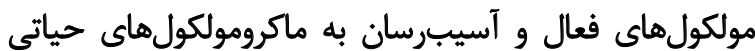

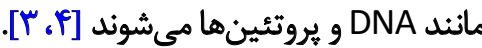

امروزه به علت سميت، عوارض و هزينه نسبتاً زياد توليد

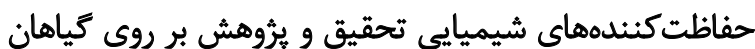

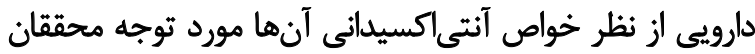

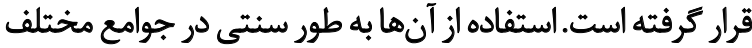

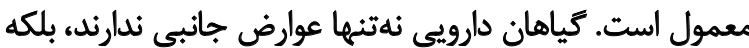

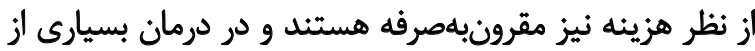

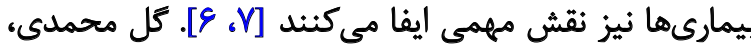

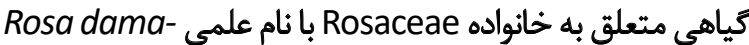

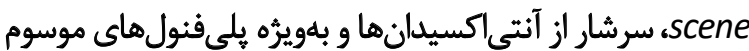

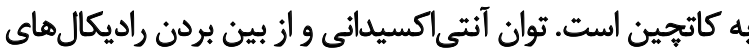

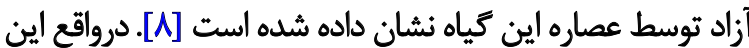

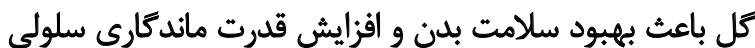

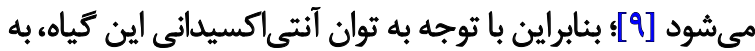

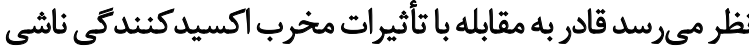

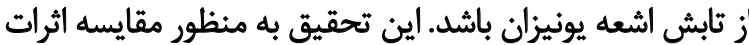

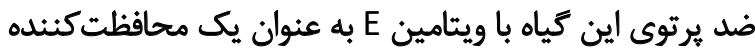
يرثوى شناختهشده انجام شده است.

مواد وروشها تها

اين تحقيق يك مطالعه آزمايشعاهى است كه بر روك روى خون

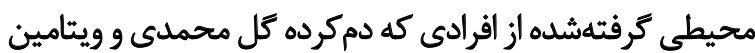

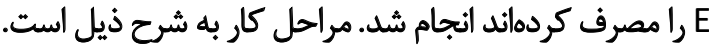

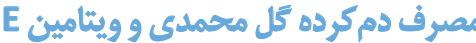
از بين داوطلبينى كه يرسشنامه مربوط به سبك زئدكى و وردي

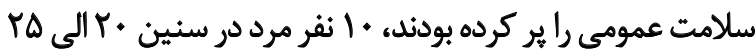

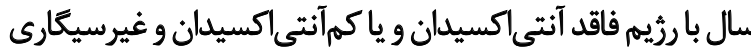

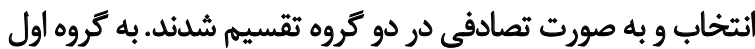

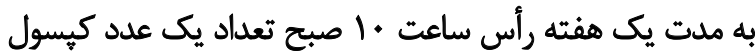

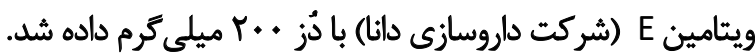

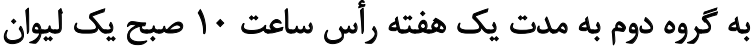

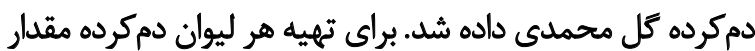

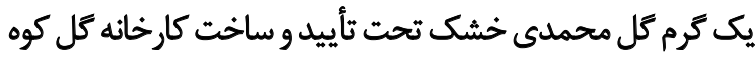




\section{مصرف ويتامين}

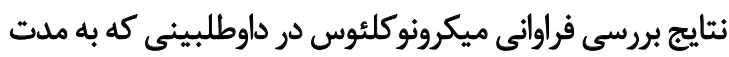

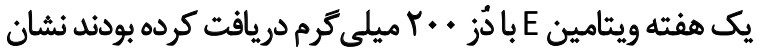

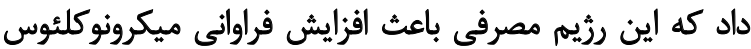

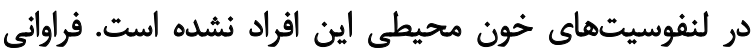

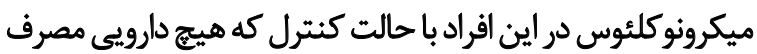
نكردهاند تفاوت معنى دارى را نشان نمى دهد (تصوير شماره الئ ).

\section{مصرف دم كره تحل محمدي}

نتايج مصرف يك هفته دمكرده كل محمدى بر افزايش فراوانى

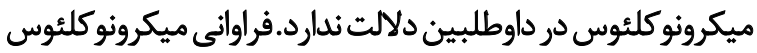
در اين افراد تفاوت معنىدارى را بادين حالت كنترل نشان ندان نداد

(تصوير شماره ب).

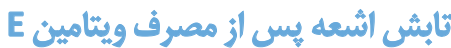

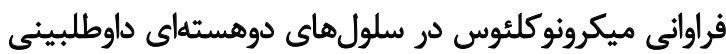

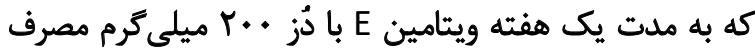

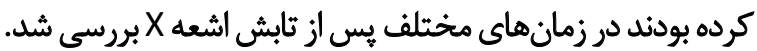

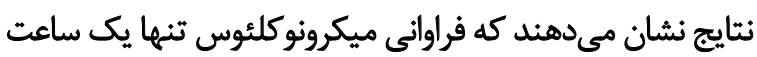

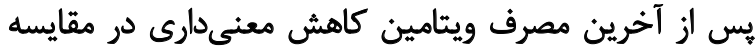

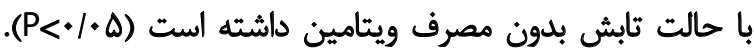

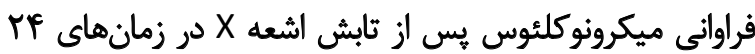

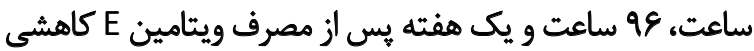

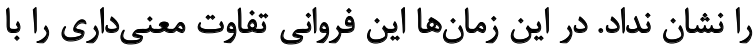
حالت تابش اشعه بدون مصرف ويتامين ندارد (تصوير شماره سا).

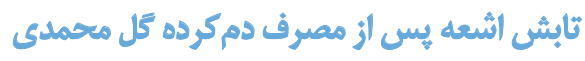

مصرف دمكرده كل محمدى به مدات يك هفته باعث كاهش

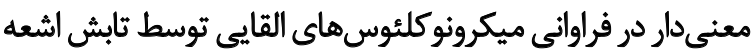

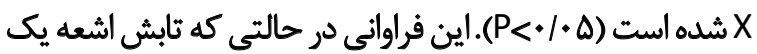

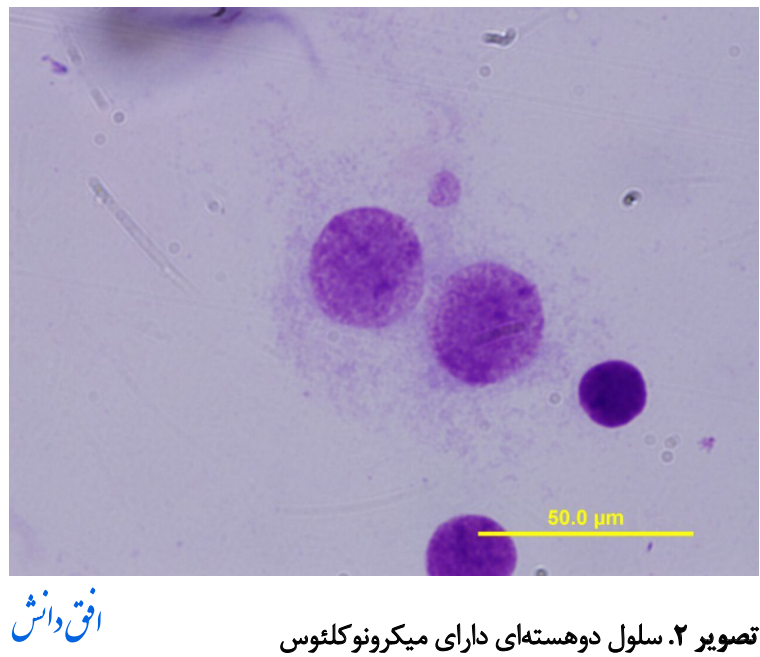

.1

$$
\begin{aligned}
& \text { تعداد سلول هاى دوهستهاى }
\end{aligned}
$$

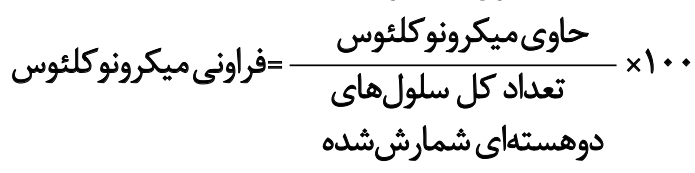

آناليثز آمارى

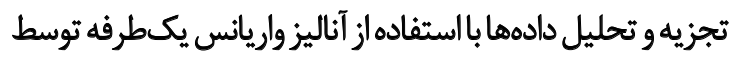

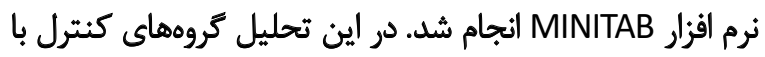

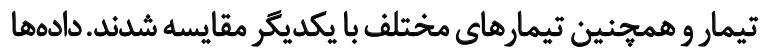

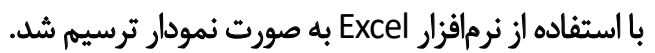

ياثتهنه

استفاده از سيتوكالاسين b باعث توقف سيتوكينز و ايجاد

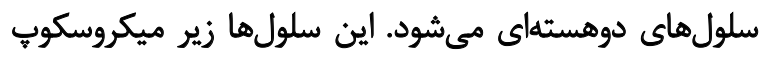

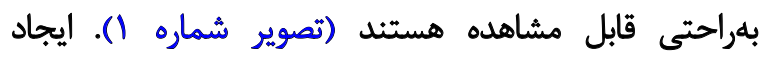

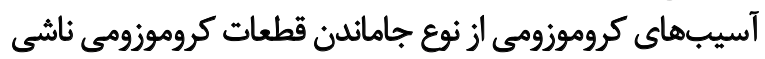

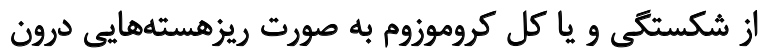

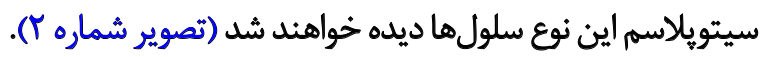

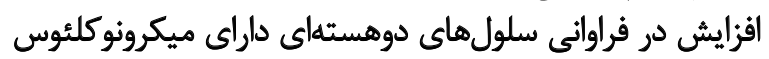
بيانكًر افزايش صدمات كروموزومى است.

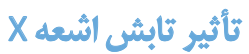

تابش اشعه ايكس با ذز 2Gy باعث افزايش معنى دار فراوانى

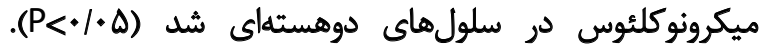

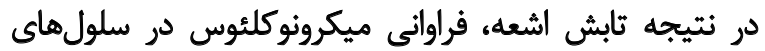

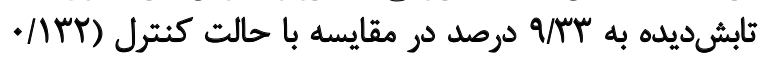
درصد) افزايش يافت (تصوير شماره (1).

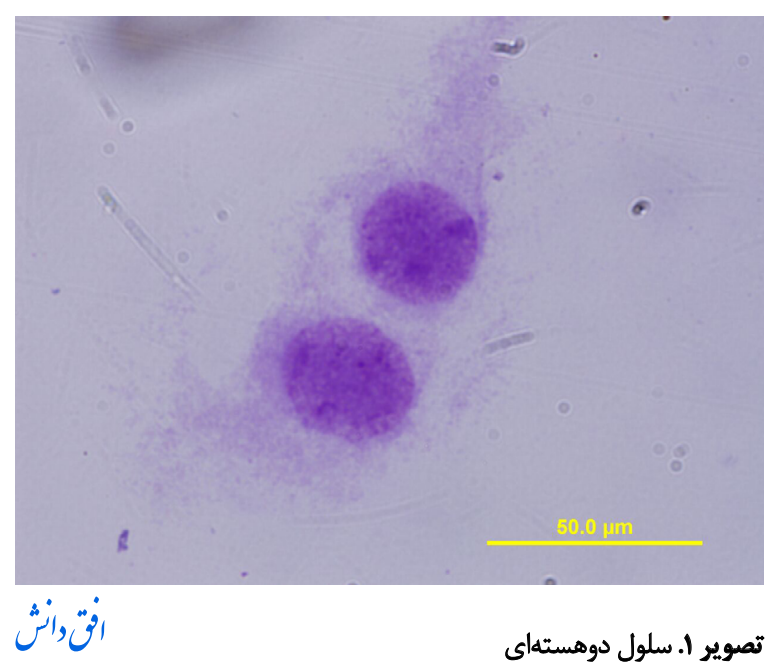


ترتيب كه قطعات كروموزومى بدون سانترومرو يا كروموزومهاى

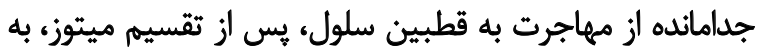

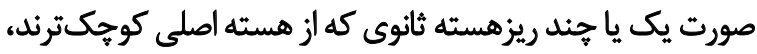

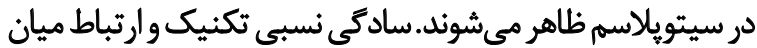

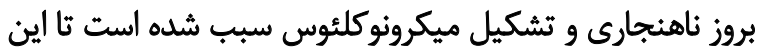

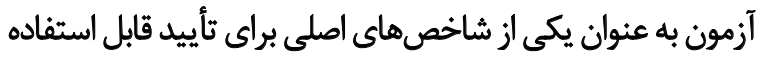

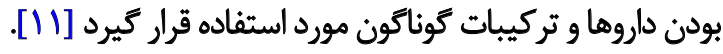

در اين تحقيق، تأثير محافظت كروموزومى ويتامين E و ودمكرده

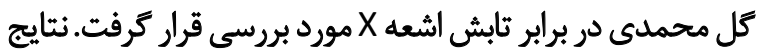

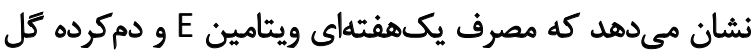

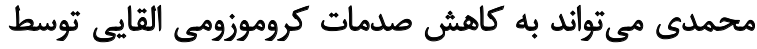

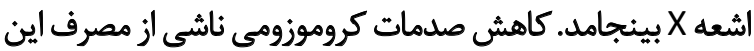

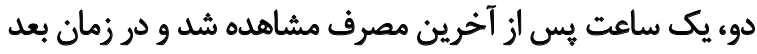

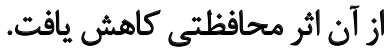

جلوكيرى از اثرات مخرب ثابش اشعه و يا كاهش اين اثرات

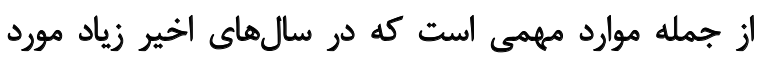

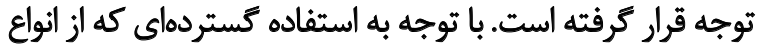

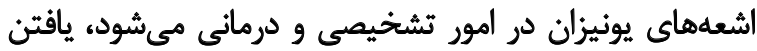

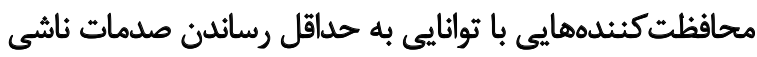

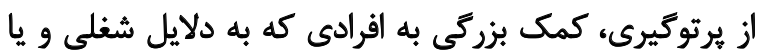

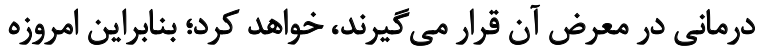

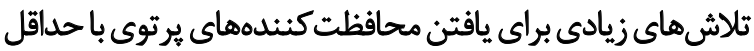

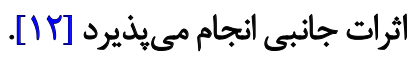

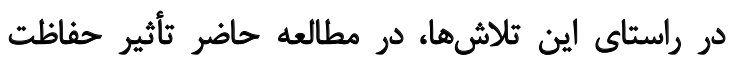

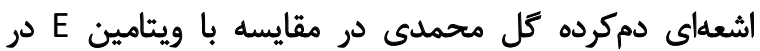

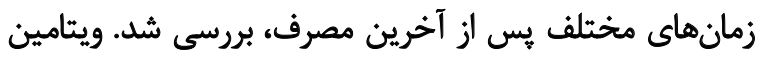

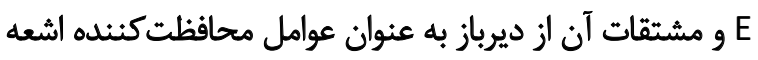

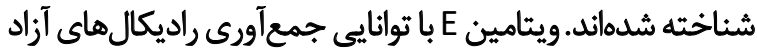

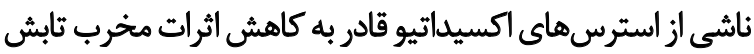

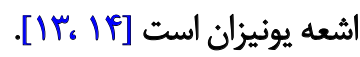

در مطالعه حاضر تابش 2Gy اشعه كاما به نمونه خون

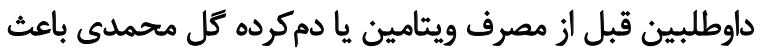

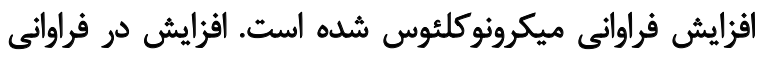

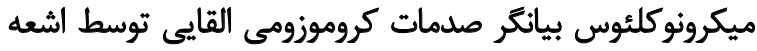

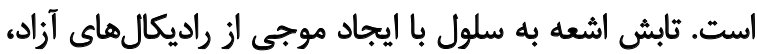

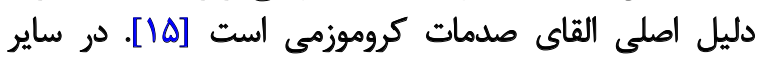
مطالعات به ميزان مشابهى با مطالعه حاضر اشعه يونئيز

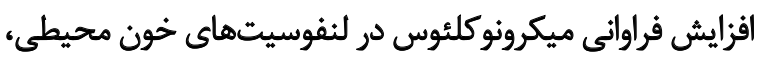

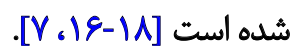

مصرف يكهفتهاى ويتامين E قادر به كاهش صدان

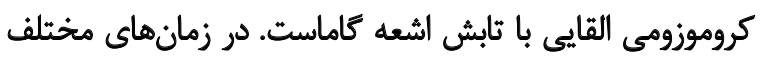

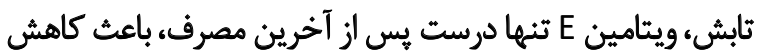

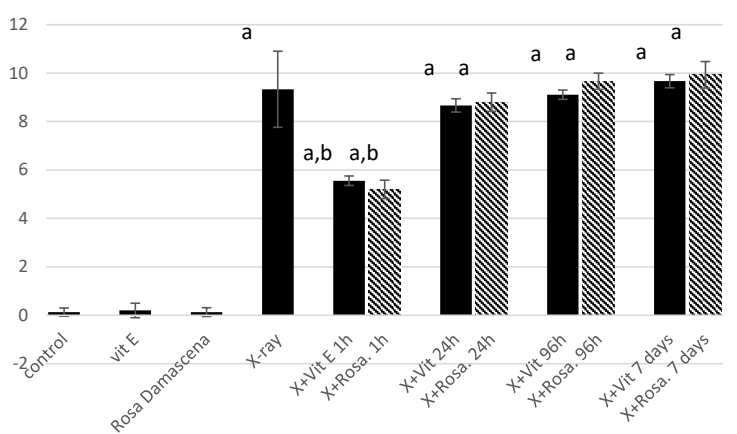

نُ

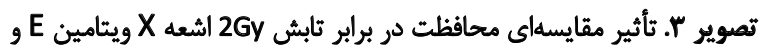
دمرده كل محمدى (Rosa Damascena)

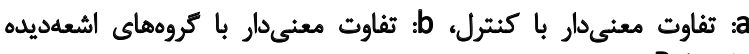
$(P<\bullet / \bullet \Delta)$

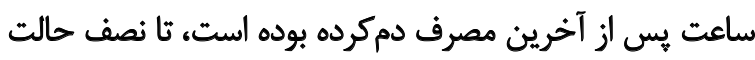

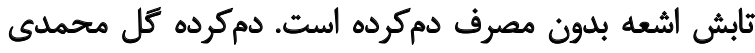

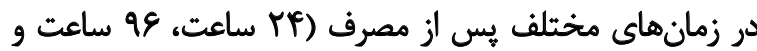

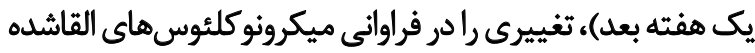

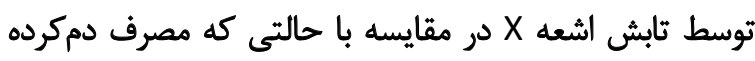
صورت نكرفتهه، باعث نشده است (تصوير شماره سَ).

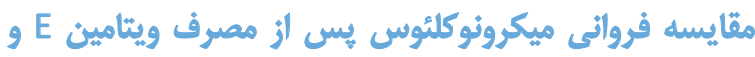

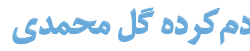

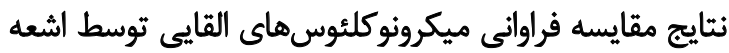

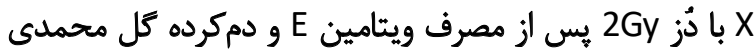

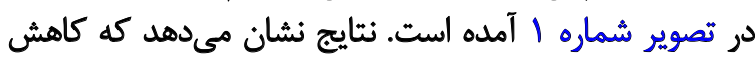

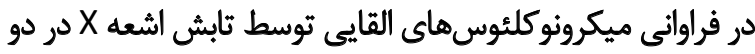

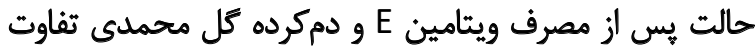

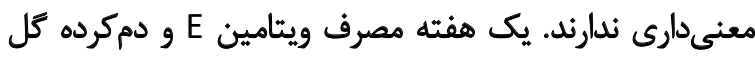

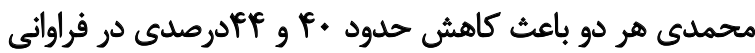

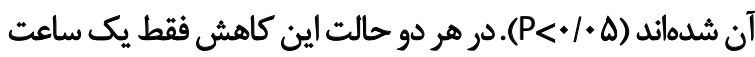

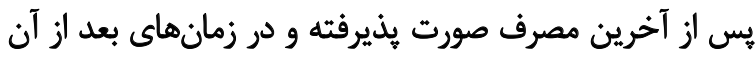

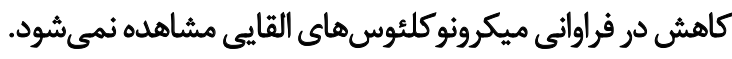

بحث

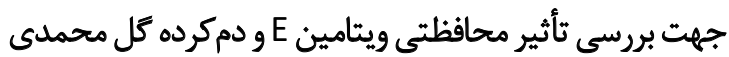

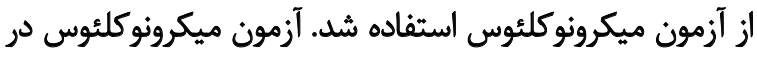

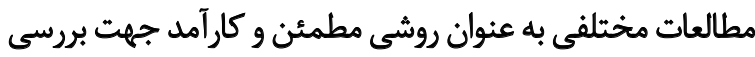

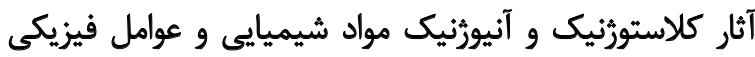

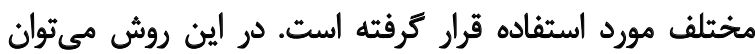

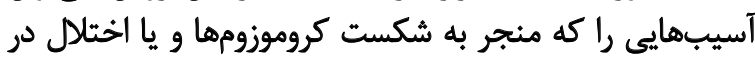
مهاجرت آنها در تقسيمات سلولى مىشود، تشخيص داد. به اين 


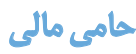 \\ اين مطالعه با هزينه شخصي انجام شده است.

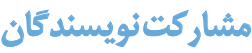

تمامى نويسندكان در آمادهازى اين مقاله مشاركت داشتهاند.

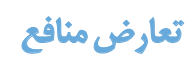

بنا به اظهار نويسندكان،اين مقاله هيجّونه تعارض منافعى ندارد.

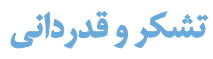

نويسندكان مقاله برخود لازم مى إنداند از همكارى صميمانه

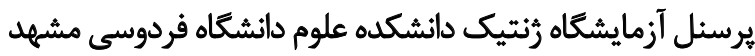

قدرداني و تشكر كنيند.
صدمات كروموزومى القايى توسط اشعه شد؛ بنابراين به نظر ميرسد

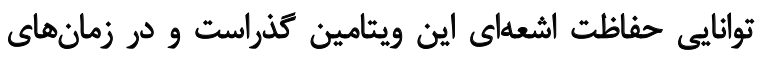

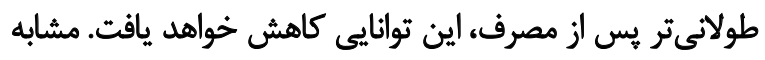

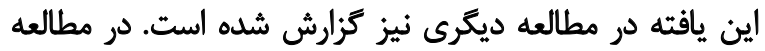

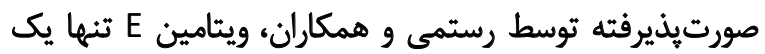

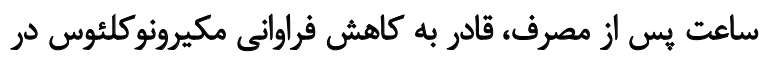

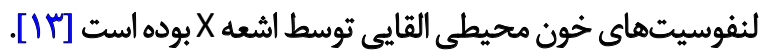

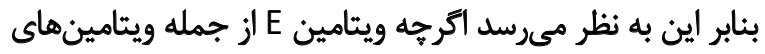

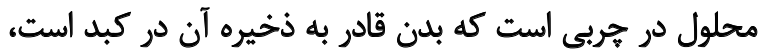

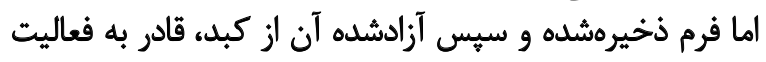

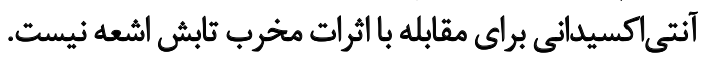
مصرف يكهفتهاى كياه كل محمدى در افراد داوطلب باعث

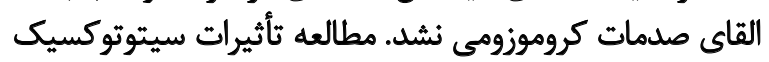

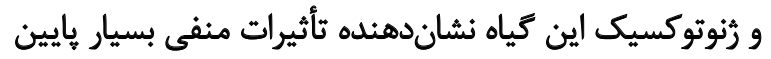

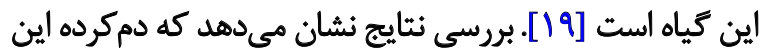

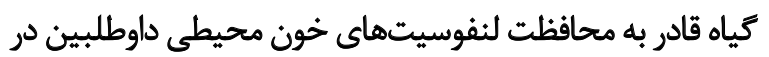

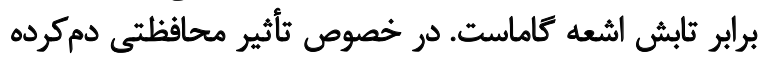

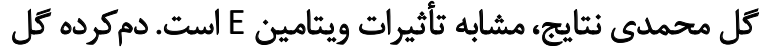

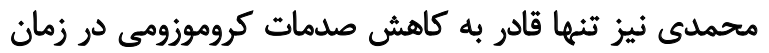

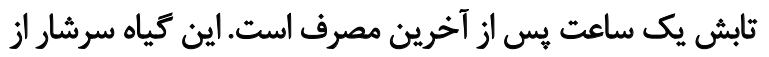

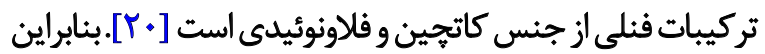

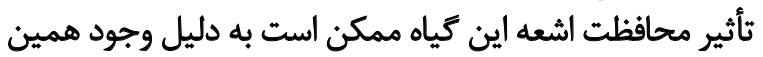

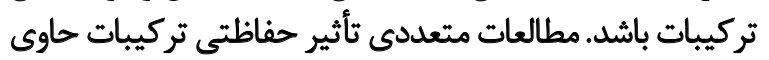

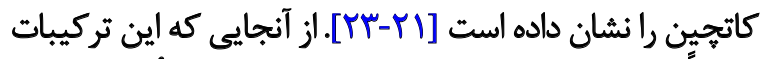

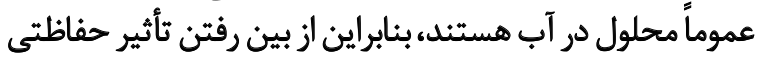

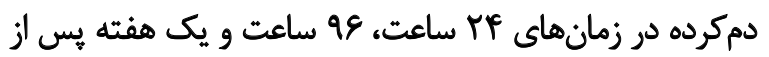
اخرين مصرف به دليل دفع تركيبات مؤثره از بدن افر براد است است

$$
\text { تشيجلميرى }
$$

نتايج مطالعه حاضر نشان ميدهد كه دمكرديه كل محمدي

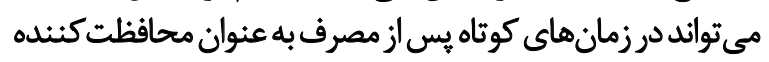

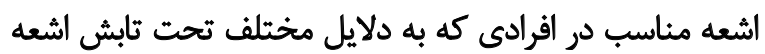

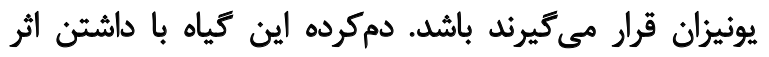

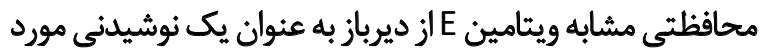

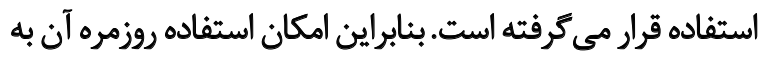

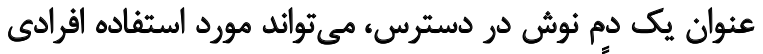

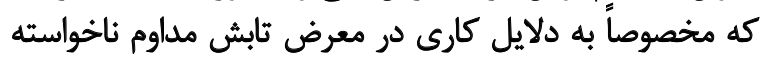

$$
\text { هستند، قرار بكيرد. }
$$

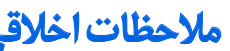

\section{مييروى ازٔ اصول انغلاث يُوهش}

انجام اين مطالعه طبق نامه تأييديه كميته اخلاق در يروهش برهاي

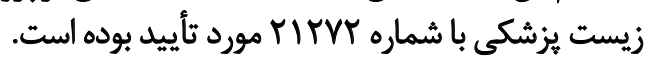




\section{References}

[1] Reisz JA, Bansal N, Qian J, Zhao W, Furdui CM. Effects of ionizing radiation on biological molecules--mechanisms of damage and emerging methods of detection. Antioxidants \& Redox Signaling. 2014; 21(2):26092. [DOI:10.1089/ars.2013.5489] [PMID] [PMCID]

[2] Thomas MJ. The role of free radicals and antioxidants. Nutrition. 2000; 16(7-8):716-8. [DOI:10.1016/S0899-9007(00)00343-9]

[3] Alkadi H. A review on free radicals and antioxidants. Infectious Disorders Drug Targets. 2020; 20(1):16-26. [DOI:10.2174/187152651866618 0628124323] [PMID]

[4] Sharma GN, Gupta G, Sharma P. A comprehensive review of free radicals, antioxidants, and their relationship with human ailments. Critical Reviews in Eukaryotic Gene Expression. 2018; 28(2):139-54. [DOI:10.1615/CritRevEukaryotGeneExpr.2018022258] [PMID]

[5] Limoli CL, Giedzinski E, Morgan WF, Swarts SG, Jones GDD, Hyun W. Persistent oxidative stress in chromosomally unstable cells. Cancer Research. 2003; 63(12):3107-11. [PMID]

[6] Kuntić VS, Stanković MB, Vujić ZB, Brborić JS, Uskoković-Marković SM. Radioprotectors - the evergreen topic. Chemistry \& Biodiversity. 2013; 10(10):1791-803. [DOI:10.1002/cbdv.201300054] [PMID]

[7] Hosseinimehr SJ. Beneficial effects of natural products on cells during ionizing radiation. Reviews on Environmental Health. 2014; 29(4):34153. [DOI:10.1515/reveh-2014-0037] [PMID]

[8] Kalim MD, Bhattacharyya D, Banerjee A, Chattopadhyay Sh. Oxidative DNA damage preventive activity and antioxidant potential of plants used in Unani system of medicine. BMC Complementary and Alternative Medicine. 2010; 10:77. [DOI:10.1186/1472-6882-10-77] [PMID] [PMCID]

[9] Jafari M, Zarban A, Pham S, Wang T. Rosa damascena decreased mortality in adult Drosophila. Journal of Medicinal Food. 2008; 11(1):9-13. [DOI:10.1089/jmf.2007.546] [PMID]

[10] Fenech M, Morley AA. Measurement of micronuclei in lymphocytes. Mutation Research/Environmental Mutagenesis and Related Subjects. 1985; 147(1-2):29-36. [DOI:10.1016/0165-1161(85)90015-9]

[11] Fenech M. In vitro micronucleus technique to predict chemosensitivity. In: Blumenthal RD, editor. Chemosensitivity: Volume II, Methods in Molecular Medicine ${ }^{\mathrm{TM}}$. Vol. 111. Totowa, NJ: Humana Press; 2005. p. 3-32. [DOI:10.1385/1-59259-889-7:003] [PMID]

[12] Smith TA, Kirkpatrick DR, Smith S, Smith TK, Pearson T, Kailasam A, et al. Radioprotective agents to prevent cellular damage due to ionizing radiation. Journal of Translational Medicine. 2017; 15:232. [DOI:10.1186/ s12967-017-1338-x] [PMID] [PMCID]

[13] Rostami A, Moosavi SA, Changizi V, Abbasian Ardakani A. Radioprotective effects of selenium and vitamin-E against $6 \mathrm{MV}$ X-rays in human blood lymphocytes by micronucleus assay. Medical Journal of the Islamic Republic of Iran. 2016; 30:367. [PMID] [PMCID]

[14] Singh VK, Beattie LA, Seed TM. Vitamin E: Tocopherols and tocotrienols as potential radiation countermeasures. Journal of Radiation Research. 2013; 54(6):973-88. [DOI:10.1093/jrr/rrt048] [PMID] [PMCID]

[15] Zhao W, Diz DI, Robbins ME. Oxidative damage pathways in relation to normal tissue injury. The British Journal of Radiology. 2007; 80(1):S23S31. [DOI:10.1259/bjr/18237646] [PMID]

[16] Hosseinimehr SJ, Nobakht R, Ghasemi A, Allahverdi Pourfallah T. Radioprotective effect of mefenamic acid against radiation-induced genotoxicity in human lymphocytes. Radiation Oncology Journal. 2015; 33(3):256-60. [DOI:10.3857/roj.2015.33.3.256] [PMID] [PMCID]

[17] Hosseinimehr SJ, Fathi M, Ghasemi A, Rezaeian Shiadeh SN, Allahverd Pourfallah T. Celecoxib mitigates genotoxicity induced by ionizing radiation in human blood lymphocytes. Res Pharm Sci. 2017; 12(1):82-7. [DOI:10.4103/1735-5362.199051] [PMID] [PMCID]

[18] Zal Z, Ghasemi A, Azizi Sh, Asgarian-Omran H, Montazeri A, Hosseinimehr SJ. Radioprotective effect of cerium oxide nanoparticles against genotoxicity induced by ionizing radiation on human lymphocytes. Current Radiopharmaceuticals. 2018; 11(2):109-15. [DOI:10.2174/1874471 011666180528095203] [PMID]

[19] Shokrzadeh M, Habibi E, Modanloo M. Cytotoxic and genotoxic studies of essential oil from Rosa damascene Mill., Kashan, Iran. Medicinski Glasnik (Zenica). 2017; 14(2):152-7. [PMID]

[20] Safari MR, Azizi O, Heidary SS, Kheiripour N, Pouyandeh Ravan AR Antiglycation and antioxidant activity of four Iranian medical plant extracts. Journal of Pharmacopuncture. 2018; 21(2):82-9. [DOI:10.3831/ KPI.2018.21.010] [PMID] [PMCID]

[21] Modak A, Chakraborty A, Das SK. Black tea extract protects against $Y$-radiation-induced membrane damage of human erythrocytes. Indian Journal of Experimental Biology. 2016; 54(11):745-52. http://nopr.niscair.res.in/handle/123456789/36896

[22] Ding J, Wang H, Wu ZB, Zhao J, Zhang S, Li W. Protection of murine spermatogenesis against ionizing radiation-induced testicular injury by a green tea polyphenol. Biology of Reproduction. 2015; 92(1):6. [DOI:10.1095/biolreprod.114.122333] [PMID]

[23] Nair CKK, Salvi VP. Protection of DNA from gamma-radiation induced strand breaks by Epicatechin. Mutation Research/Genetic Toxicology and Environmental Mutagenesis. 2008; 650(1):48-54. [DOI:10.1016/j. mrgentox.2007.10.001] [PMID] 
This Page Intentionally Left Blank 